\section{Femtosecond Phase-Coherent Two-Dimensional Spectroscopy}

\author{
Peifang Tian, ${ }^{1}$ Dorine Keusters, ${ }^{2}$ Yoshifumi Suzaki, ${ }^{3}$ \\ Warren S. Warren ${ }^{2}$
}

\begin{abstract}
Femtosecond phase-coherent two-dimensional (2D) spectroscopy has been experimentally demonstrated as the direct optical analog of 2D nuclear magnetic resonance. An acousto-optic pulse shaper created a collinear three-pulse sequence with well-controlled and variable interpulse delays and phases, which interacted with a model atomic system of rubidium vapor. The desired nonlinear polarization was selected by phase cycling (coadding experimental results obtained with different interpulse phases). This method may enhance our ability to probe the femtosecond structural dynamics of macromolecules.
\end{abstract}

Two of the central questions in physical chemistry and biophysics are how the structures of complex molecular systems (such as proteins and hydrogen-bonded liquids) evolve, and how such dynamics are related to important chemical and biological processes. Two-dimensional (2D) nuclear magnetic resonance (NMR) can resolve the structure of complex molecules with atomic resolution, but its millisecond time scale is too slow to follow ultrafast dynamics (tens of femtoseconds to picoseconds), which is crucial to understanding many chemical and biological processes (1-3). Both ultrafast electronic and vibrational 2D spectroscopy have recently shown promise for use in probing the structures and dynamics of systems such as laser dyes, metal carbonyls, peptides, and proteins (3-16). However, these techniques use noncollinear pulses to average over phase in a spatially coherent manner, rather than collinear phasecoherent pulses, which are pulses with well-defined interpulse phases as in NMR (17). The latter approach, the direct optical analog of NMR, can provide advantages over existing techniques for extracting spectral information. We demonstrated experimentally that it is technically possible. This direct optical analog of 2D NMR, combined with current 2D spectroscopic methods, can enhance capabilities to resolve the ultrafast structural dynamics of complex systems.

In current ultrafast $2 \mathrm{D}$ experiments, a molecular system interacts with three ultrafast pulses traveling along different directions with wave vectors $\boldsymbol{k}_{\mathbf{1}}, \boldsymbol{k}_{\mathbf{2}}$, and $\boldsymbol{k}_{\mathbf{3}}$, respectively (Fig. 1A) (4-9). The first pulse prepares the system in a particular coher-

${ }^{1}$ Department of Electrical Engineering, ${ }^{2}$ Department of Chemistry, Center of Ultrafast Laser Applications, Princeton University, Princeton, NJ 08544, USA. ${ }^{3}$ Department of Advanced Materials Science, Faculty of Engineering, Kagawa University, Takamatsu, Kagawa 761-0396, Japan. ence, which oscillates during the period $\tau_{1}$. The second and third pulses interrupt this oscillation and create a new coherence. The complete polarization $P$ is then detected at a delay of $\tau_{2}$ after the third pulse and as a function of delay $\tau_{1}$. A $2 \mathrm{D}$ function $P\left(\tau_{1}, \tau_{2}\right)$ is thus obtained and Fourier-transformed to create a $2 \mathrm{D}$ spectrum $S\left(f_{1}, f_{2}\right)$ (4-9). The experimental 2D spectral features (peak positions, line shapes, and polarization dependence) reveal the properties of the molecular system (transition frequencies, coupling, anharmonicity, and interactions with the environment) from which structural information can be deduced (3-16). Structural dynamics can be monitored by taking a series of 2D spectra $S\left(f_{1}, f_{2}\right)$ as a function of waiting time $T$ between the second and third pulses (4-12).

Different interactions between the molecular system and the three pulses generate many peaks in the $2 \mathrm{D}$ spectrum $S\left(f_{1}, f_{2}\right)$ (17). Structural information can often be obtained from just a few peaks caused by particular (and often weak) third-order nonlinear polarizations, such as the subject of this experiment: the photon echo signal $P_{\text {echo }}^{(3)}$. Other peaks only give redundant information and congest the spectrum. In current ultrafast $2 \mathrm{D}$ spectroscopy, $P_{\text {echo }}^{(3)}$ is isolated by measuring it in a unique direction $\boldsymbol{k}_{s}=\boldsymbol{k}_{3}+\boldsymbol{k}_{\mathbf{2}}-\boldsymbol{k}_{\mathbf{1}}$ determined by the phase-matching condition (Fig. 1A) (4-9). The phase information on $P_{\text {echo }}^{(3)}$ is obtained with heterodyne detection. Phase matching has the advantage of spatial separation of the desired and undesired signals.

However, $P_{\text {echo }}^{(3)}$ also has a unique phase dependence on the input pulses. Therefore, when collinear phase-coherent pulses interact with the molecular system, $P_{\text {echo }}^{(3)}$ can be selected by its phase dependence, leading to the direct optical analog of 2D NMR: ultrafast 2D spectroscopy with phase cycling (17). In this approach, phase information on $P_{\text {echo }}^{(3)}$ is obtained by phase cycling (altering the inter- pulse phase shifts in a controlled manner). Phase cycling has several advantages over phase matching. Pulses with specific controlled phases can alter the effective molecular evolution, thus coherently averaging away specific interaction mechanisms or enhancing signal intensity $(1,2,17)$. The collinear approach also conveniently permits data acquisition in a "rotating frame" that dramatically decreases the number of required data points. In addition, noncollinear phase matching only works in extended systems (many absorbers with spatial distribution $\gg 1 / \Delta k$, where $\Delta k$ is the difference between the wave vectors of the polarization and its corresponding electric field), whereas the collinear phase cycling is not constrained by the size of the sample. Finally, the complexity of the phase-matching experiment increases rapidly with the addition of extra pulses coming from different directions.

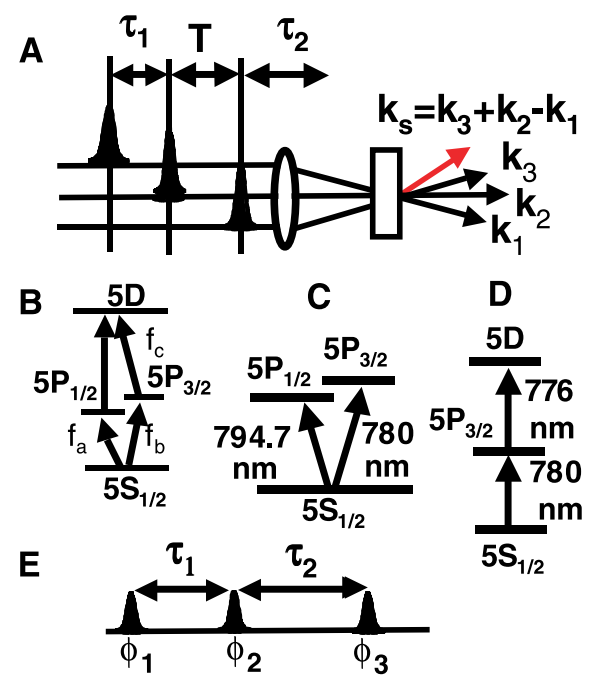

Fig. 1. (A) Pulse sequence used in ultrafast 2D spectroscopy with phase matching. Three excitation pulses, separated by delays $\tau_{1}$ and $T$ and traveling in different directions $\boldsymbol{k}_{1}, \boldsymbol{k}_{\mathbf{2}}$, and $\boldsymbol{k}_{\mathbf{3}}$, respectively, are focused onto the sample. The photon echo signal $P_{\text {echo }}^{(3)}$ is emitted in a different direction $\boldsymbol{k}_{\mathrm{s}}=\boldsymbol{k}_{\mathbf{3}}+\boldsymbol{k}_{\mathbf{2}}-\boldsymbol{k}_{\mathbf{1}}$ and hence is spatially isolated. $P_{\text {echo }}^{(3)}$ is measured as a function of $\tau_{1}$ and $\tau_{2}$. (B) Schematic diagram of the lowest four energy levels of $\mathrm{Rb}$ atoms relevant to our experiment: ground state $5 S_{1 / 2}$, two singly excited states $5 P_{1 / 2}$ and $5 P_{3 / 2}$, and one doubly excited state $5 D$. (C) System I: $D_{1}$ and $\mathrm{D}_{2}$ transitions share the same ground state (absorption wavelength $\lambda$ at 794.7 and $780 \mathrm{~nm}$, respectively). (D) System II, with one ground $\left(5 S_{1 / 2}\right)$, one singly $\left(5 P_{3 / 2}\right)$ excited, and one doubly $(5 D)$ excited state (corresponding to two transitions at $\lambda=780$ and $776 \mathrm{~nm}$, respectively), is used to simulate anharmonicity. (E) Pulse sequence used in ultrafast 2D spectroscopy with phase cycling. Three collinear pulses, with phases $\phi_{1}, \phi_{2}$, and $\phi_{3}$, are separated by delays of $\tau_{1}$ and $\tau_{2}$. This pulse sequence is equivalent to that of $(A)$ by setting $T=0$ and including a third pulse to detect the polarization. 


\section{R E P O R T S}

Optical 2D spectroscopy with phase cycling was proposed in 1976 (1), and complex phase-cycled sequences were demonstrated with nanosecond resolution in 1981 (18). Recent developments in ultrafast pulse-shaping technologies [such as the acousto-optic pulse shaper (AOPS) and liquid-crystal-based pulse shaper (LCPS)] and highly stable amplified ultrafast laser systems now make it possible to create arbitrarily complex femtosecond pulse sequences (19-21). In fact, ultrafast 1D spectroscopy with phase cycling was demonstrated by creating a phasecoherent pulse pair by using either an AOPS or a complicated feedback loop $(21,22)$.

We generated a phase-coherent threepulse sequence using an AOPS and demonstrated ultrafast 2D spectroscopy using phase cycling on a model system: Rubidium $(\mathrm{Rb})$ atomic vapor. $\mathrm{Rb}$ atoms can be used to model two crucial properties: coupling and anharmonicity of a molecular system, which manifest themselves as cross peaks $\left(f_{1} \neq \pm f_{2}\right)$ on a $2 \mathrm{D}$ spectrum (17). The lowest four energy levels relevant to our experiment (Fig. 1B) are the ground state $5 S_{1 / 2}$, two singly excited states $5 P_{1 / 2}$ and $5 P_{3 / 2}$, and one doubly excited state $5 D$. The three relevant transitions are $D_{1}: 5 S_{1 / 2} \rightarrow 5 P_{1 / 2} ; \mathrm{D}_{2}: 5 S_{1 / 2} \rightarrow 5 P_{3 / 2}$; and $5 P_{3 / 2} \rightarrow 5 D$, with transition frequencies of $f_{a}$, $f_{b}$, and $f_{c}$, respectively. The transition $5 P_{1 / 2}$ $\rightarrow 5 D$ was not directly excited in our experiment because of its small transition dipole moment. Depending on the input pulse spectrum, two systems can be obtained. System I (Fig. 1C) is a three-level "V" system, where the two transitions $D_{1}$ and $D_{2}$ share the same ground state, allowing us to simulate coupling. System II (Fig. 1D) with one ground, one singly excited, and one doubly excited state simulates anharmonicity. We can switch between these two systems by controlling the frequency content of the laser pulses.

Three collinear pulses with delays $\tau_{1}$ and $\tau_{2}$ were used (Fig. 1E). The first and second pulse are the excitation pulses and serve the same role as the three pulses in Fig. 1A with $T=0$. The phase and amplitude of the ultrafast oscillating polarization need to be measured as a function of $\tau_{1}$ and $\tau_{2}$, which is difficult to achieve directly. Therefore, an additional (third) pulse is added at delay $\tau_{2}$ after the second pulse. This third pulse converts the polarization at delay $\tau_{2}$ into a population of the excited states (18). This population reflects the amplitude and phase of the polarization at $\tau_{2}$ and is thus related to the third-order polarization. Although the most common method of magnetization detection in NMR is nuclear induction (directly observing the oscillating magnetization), detection directly analogous to our method is done in magnetic resonance experiments such as force microscopy. Hence, the complete polarization can be obtained by measuring the population as a function of $\tau_{1}$ and $\tau_{2}$, which can be done by measuring the fluorescence from or absorption by $\mathrm{Rb}$ atoms. Another alternative, which would probably be very useful for infrared (IR) pulse sequences to study multilevel vibrational systems, is to apply a final laser pulse at a wavelength $(\lambda)$ that is more strongly absorbed by a vibrationally excited molecule than by a groundstate molecule. For example, for a $2000 \mathrm{~cm}^{-1}$ vibrational frequency, we may go to the red end of the ultraviolet (UV) absorption spectrum and thus look at changes in UV-visible fluorescence with phase cycling.

The total electric field $E(t)$ can be written as $\mathrm{E}(t)=\left[A\left(t+\tau_{1}\right) e^{-i\left(2 \pi f_{0} t-\phi_{1}\right)}+A(t) e^{-i\left(2 \pi f_{0} t-\phi_{2}\right)}\right.$ $\left.+A\left(t-\tau_{2}\right) e^{-i\left(2 \pi f_{0} t-\phi_{3}\right)}\right]+$ a complex conjugate where $A(t)$ and $f_{0}$ are the envelope and central frequency of each pulse, and $\phi_{1}, \phi_{2}$, and $\phi_{3}$ are the phases of pulses 1,2, and 3, respectively.

In the system I case, the population $\rho$ of $5 P_{1 / 2}$ and $5 P_{3 / 2}$ after the three pulses is

$$
\sum_{i=1}^{36} a_{i} c_{i}\left(\phi_{1}, \phi_{2}, \phi_{3}\right) e^{i 2 \pi f_{1} \tau_{1}} e^{i 2 \pi f_{2} \tau_{2}}
$$

where each term corresponds to a particular polarization and thus to a unique peak in the 2D spectrum (17). The position, amplitude, and phase dependence of each peak are $\left(f_{1}\right.$, $\left.f_{2}\right), a_{i}$, and $c_{i}\left(\phi_{1}, \phi_{2}, \phi_{3}\right)$, respectively. The photon echo signal includes two antidiagonal peaks at $\left(\Delta f_{a},-\Delta f_{a}\right)$ and $\left(\Delta f_{b},-\Delta f_{b}\right)$ and two cross peaks $\left(\Delta f_{a},-\Delta f_{b}\right)$ and $\left(\Delta f_{b},-\Delta f_{a}\right)$ with the same phase dependence $\left.e^{\mathrm{i}\left(-\phi_{1}\right.}+2 \phi_{2}-\phi_{3}\right)$ (17). $\Delta f_{a}=f_{a}-f_{\mathrm{L}}$ and $\Delta f_{b}=f_{b}-f_{\mathrm{L}}$ are the difference frequencies between the transition frequencies and the locked frequency $f_{\mathrm{L}}$ of the laser (23). Hence, the phase-cycling scheme systematically reduces the oscillation frequency and allows a reduction in the number of sampling points in the time domain $(17,21,22)$.

The photon echo peaks can be understood as follows. The first pulse excites coherences $D_{1}$ and $D_{2}$ during $\tau_{1}$. Two interactions with the second pulse can reverse the phase of the polarization, leading to an antidiagonal peak at $\left(\Delta f_{a},-\Delta f_{a}\right)$, or convert a $D_{1}$ coherence into a coherence at $D_{2}$, resulting in a cross peak at $\left(\Delta f_{a},-\Delta f_{b}\right)$. Similarly, two other peaks appear for those atoms oscillating at $D_{2}$ during $\tau_{1}$. The phases that the photon echo peaks acquire during $\tau_{1}$ are conjugate with those obtained during $\tau_{2}$. For example, the antidiagonal peak $\left(\Delta f_{a},-\Delta f_{a}\right)$ has phases $e^{i 2 \pi \Delta f_{a} \tau_{1}}$ and $e^{-i 2 \pi \Delta f_{a} \tau_{2}}$. This rephasing property makes the photon echo signal useful in probing the dynamics in the condensed phases. In general, cross peaks reflect the couplings between chromophores and thus provide useful structural information (9).

The phase dependence of the photon echo signal $e^{i\left(-\phi_{1}+2 \phi_{2}-\phi_{3}\right)}$ is different from those of other peaks in the 2D spectrum. For example, the peak at $(0,0)$ is caused by the linear absorption of each individual pulse and does not depend on pulse phase. To eliminate such congesting peaks, we take two sets of data for two phase combinations $\phi_{1}, \phi_{2}, \phi_{3}$ being $X X X$ and $X X \bar{X}$, respectively, and record the corresponding total populations as $\rho_{X X X}\left(\tau_{1}, \tau_{2}\right)$ and $\rho_{X X \bar{X}}\left(\tau_{1}, \tau_{2}\right)$, where symbols $X, Y, \bar{X}$, and $\bar{Y}$ represent a phase of $0, \pi / 2, \pi$, and $3 \pi / 2$, respectively. The amplitude of the photon echo peaks is doubled in the $2 \mathrm{D}$ spectrum of the two-step phase-cycled signal $\rho 2=$ $\rho_{X X X}-\rho_{X X \bar{X}}$, whereas the DC peak disappears along with all of the peaks that do not depend on the phase of the third pulse (17).

Populations for 16 different phase combinations of the three pulses can be recorded and then combined correctly to select the photon echo peaks while completely eliminating all of the other peaks. For example, the photon echo peaks can be selected by the following 16-step phase cycle (17)

$$
\begin{aligned}
\rho 16= & \left(\rho_{X X X}-\rho_{X X \bar{X}}-\rho_{\bar{X} X X}+\rho_{\bar{X} X \bar{X}}-\rho_{Y X Y}+\right. \\
& \left.\rho_{\bar{Y} X Y}+\rho_{Y X \bar{Y}}-\rho_{\bar{Y} X \bar{Y}}\right)+ \\
& i\left(\rho_{\bar{X} X \bar{Y}}-\rho_{\bar{X} X Y}+\rho_{X X Y}-\rho_{X X \bar{Y}}+\rho_{Y X X}\right. \\
& \left.-\rho_{Y X \bar{X}}-\rho_{\bar{Y} X X}+\rho_{\bar{Y} X \bar{X}}\right)
\end{aligned}
$$

We experimentally realized this approach by sending an amplified pulse train (at $9 \mu \mathrm{J}$ and

A

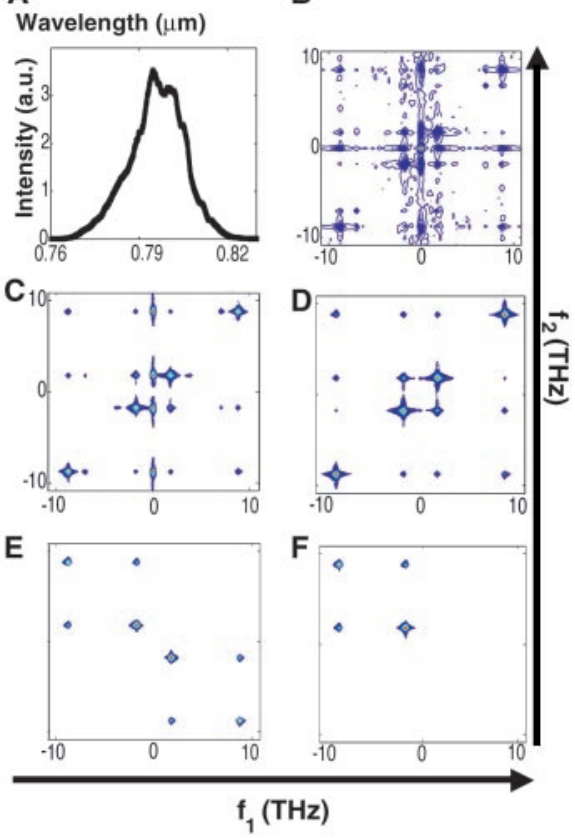

Fig. 2. (A) Pulse spectrum. (B to F) 2D spectra of system I obtained from the 2D Fourier transformation of the fluorescence signal (B) without phase cycling and with (C) 2-, (D) 4-, (E) 8-, and (F) 16-step phase cycling. Only photon echo peaks are retained after a 16-step phase cycle, as expected $\left(\Delta f_{a}=-9.1 \mathrm{THz}\right.$ and $\left.\Delta f_{b}=-2.1 \mathrm{THz}\right)$. 
a $20-\mathrm{kHz}$ repetition rate, generated by a Coherent RegA 9000 Ti:sapphire regenerative amplifier) into an AOPS. For each input pulse, a phase-coherent three-pulse sequence was then generated, with the pulse spectrum (Fig. 2A) $\lambda \leq 776 \mathrm{~nm}$ blocked to obtain system I $(20,21)$. The pulse sequence was then focused into a spot size of $\sim 100 \mu \mathrm{m}$ with $20 \mathrm{~nJ}$ per pulse and a pulse duration of $70 \mathrm{fs}$ in a $\mathrm{Rb}$ vapor cell (2-mm path length, $\sim 100^{\circ} \mathrm{C}$ ). The fluorescence perpendicular to the input beam was collected by a photomultiplier tube (PMT) (Hamamatsu R928). The signal from the PMT was amplified and then measured by a boxcar integrator (Stanford Research SR250). Each data point was an average of 3000 laser shots, although much faster updating would be possible with a more sophisticated arbitrary waveform generator.

Data with a 16-step phase cycle were collected as follows. We fixed the position and phase of pulse $2\left(\phi_{2}=0^{\circ}\right)$ and scanned $\tau_{1}, \tau_{2}$ (from 0.14 to $2.94 \mathrm{ps}$ with a step size of $46 \mathrm{fs}), \phi_{1}$, and $\phi_{3}$, respectively. At each value of $\left(\tau_{1}, \tau_{2}\right)$, the total fluorescence for all 16 different combinations of the phases $\phi_{1}$, $\phi_{2}=0$, and $\phi_{3}$ was recorded as $\rho_{i}\left(\tau_{1}, \tau_{2}\right)$, where the index $i=1,2, \ldots, 16$ represents a unique phase combination (24). Figure 2, B to $F$, show the spectra obtained without phase cycling (Fig. 2B) and with 2-, 4- $(\rho 4=\rho 2-$ $\rho \bar{X} X X+\rho \bar{X} X \bar{X}), 8-\left(\rho 8=\rho 4-\rho_{Y X Y}+\rho_{\bar{Y} X Y}\right.$ $\left.+\rho_{Y X \bar{Y}}-\rho_{\bar{Y} X \bar{Y}}\right)$, and 16-step phase-cycled signals, respectively (Fig. 2, C to F), where about half of the extra peaks are removed with each extra step. The spectrum with the 16 -step phase cycle clearly shows all photon echo peaks with $\Delta f_{a}=-9.1 \mathrm{THz}$ and $\Delta f_{b}=$ $-2.1 \mathrm{THz}$. The cross peaks arise because the $D_{1}$ and $D_{2}$ transitions share the same ground state. The results here further imply that structural information such as coupling can be clearly revealed by the phase-cycled spectrum.

Next, system II was obtained by blocking the spectrum near $794.7 \mathrm{~nm}$. Because $\Delta f_{b} \neq$ $\Delta f_{c}$, this three-level system can be treated as

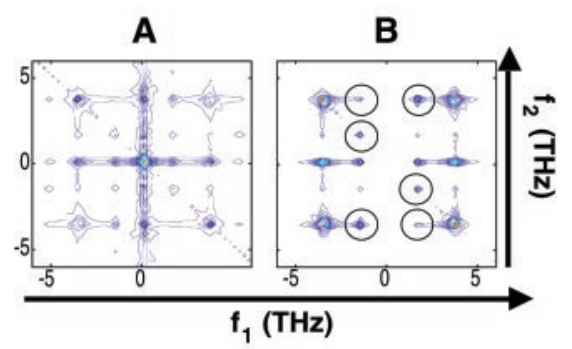

Fig. 3. 2D spectra of system II obtained from the 2D Fourier transformation of the fluorescence signal (A) without phase cycling and (B) with a two-step phase cycle $\left(\Delta f_{b}=-3.7 \mathrm{THz}\right.$ and $\Delta f_{c}=-1.6 \mathrm{THz}$ ). Spurious peaks along $f_{2}=$ $-f_{1}$ indicate that this measurement is noisy. a model for an anharmonic ladder. When the pulse sequence with phase combination $X X X$ interacts with system II, many peaks appear in the $2 \mathrm{D}$ spectrum $S\left(f_{1}, f_{2}\right)$; only peaks with the same phase dependence $e^{i\left(-\phi_{1}+2 \phi_{2}-\phi_{3}\right)}$ such as $\left(\Delta f_{b},-\Delta f_{b}\right)$ and $\left(\Delta f_{b},-\Delta f_{c}\right)$ would remain after a 16-step phase cycle, where $\Delta f_{b}$ $=-3.7 \mathrm{THz}$ and $\Delta f_{c}=-1.6 \mathrm{THz}$. Here the pulse energy was increased to $\sim 100 \mathrm{~nJ}$ to reveal the weak peak $\left(\Delta f_{b},-\Delta f_{c}\right)$. The spectra obtained from $\rho_{X X X}$ (Fig. 3A) and $\rho 2=\rho_{X X X}$ $-\rho_{\bar{X} X X}$ (Fig. 3B) both show peak $\left(\Delta f_{b},-\Delta f_{c}\right)$, as expected. The six circled peaks are caused by fifth-order polarizations and cascaded third-order signals. Two of these peaks [ $\left(\Delta f_{c}\right.$, $\left.-\Delta f_{b}\right)$ and $\left.\left(\Delta f_{c},-\Delta f_{c}\right)\right]$ have the same phase dependence as the photon echo and would remain in the $2 \mathrm{D}$ spectrum after a 16-step phase cycle. However, their amplitudes increase faster with the intensity of the input pulses than that of $\left(\Delta f_{b},-\Delta f_{c}\right)$ caused by a third-order polarization. This property may be used to eliminate or reduce the higher order peaks.

We demonstrated femtosecond phasecoherent 2D spectroscopy with a simple pulse sequence and on a model system. Because of the nonidealities of the AOPS, spurious pulses were generated along with the main pulses. Some spurious pulses overlapped with the main pulses in the time domain, which induced an amplitude modulation with the same phase dependence as the desired signal, such as $P_{\text {echo }}^{(3)}$ This problem may be solved by precompensating the spurious pulses or by replacing AOPS with LCPS. Furthermore, the total delay achievable was often constrained by either the AOPS or LCPS to about tens of picoseconds. In order to apply the phase-cycling method to macromolecules such as proteins, we need high-quality phase-coherent sequences with multiple (three or more) pulses in the mid-IR and with arbitrary polarizations. As many as hundreds of pulses can be generated with an AOPS (21). Recently, linear polarized midIR shaped pulses have been demonstrated by optical parametric amplification (25). This method can be extended to make two pulse sequences with orthogonal linear polarization, which can then be combined to yield an arbitrarily (but well-controlled) polarized phase-coherent pulse sequence (26). Therefore, complex molecular systems may soon be studied with phase cycling. Furthermore, ultrafast laser spectroscopy provides an opportunity to combine both phase cycling and phase matching (27), which may result in further simplification of the pulse sequence and allow us to detect more complicated processes.

\section{References and Notes}

1. W. P. Aue, E. Bartholdi, R. R. Ernst, J. Chem. Phys. 64, 2229 (1976).

2. R. R. Ernst, G. Bodenhausen, A. Wokaun, Principles of
Nuclear Magnetic Resonance in One and Two Dimensions (Oxford Univ. Press, Oxford, 1987).

3. Chem. Phys. 266, 135 (2001).

4. J. D. Hybl, A. A. Ferro, D. M. Jonas, J. Chem. Phys. 115, 6606 (2001).

5. M. C. Asplund, M. T. Zanni, R. M. Hochstrasser, Proc. Natl. Acad. Sci. U.S.A. 97, 8219 (2000).

6. O. Golonzka, M. Khalil, N. Demirdöven, A. Tokmakoff, Phys. Rev. Lett. 86, 2154 (2001).

7. M. T. Zanni, N.-H. Ge, Y. S. Kim, R. M. Hochstrasser, Proc. Natl. Acad. Sci. U.S.A. 98, 11265 (2001).

8. N.-H. Ge, R. M. Hochstrasser, Phys. Chem. Commun. 3, 1 (2002).

9. M. Khalil, N. Demirdoven, A. Tokmakoff, J. Phys. Chem. $A$, in press.

10. P. Hamm, M. Lim, R. M. Hochstrasser, J. Phys. Chem. B 102, 6123 (1998).

11. S. Woutersen, Y. Mu, G. Stock, P. Hamm, Proc. Natl. Acad. Sci. U.S.A. 98, 11254 (2001).

12. S. Woutersen, P. Hamm, J. Phys. Condens. Matter 14, R1035 (2002).

13. K. A. Merchant, D. E. Thompson, M. D. Fayer, Phys. Rev. Lett. 86, 3899 (2001).

14. C. Scheurer, S. Mukamel, J. Chem. Phys. 115, 4989 (2001).

15. S. Mukamel, Annu. Rev. Phys. Chem. 51, 691 (2000).

16. S. Mukamel, Principles of Nonlinear Optical Spectroscopy (Oxford Univ. Press, Oxford, 1995).

17. D. Keusters, H.-S. Tan, W. S. Warren, J. Phys. Chem. A 103, 10369 (1999).

18. W. S. Warren, A. H. Zewail, J. Chem. Phys. 75, 5956 (1981).

19. A. M. Weiner, Rev. Sci. Instrum. 71, 1929 (2000).

20. W. G. Yang, D. Keusters, D. Goswami, W. S. Warren, Opt. Lett. 23, 1843 (1998)

21. D. Keusters, P. Tian, W. S. Warren, in Ultrafast Phenomena XII, T. Elsaesser, S. Mukamel, M. M. Murnane, N.F. Scherer, Eds. (Springer, Berlin, 2001), Pp. 165167.

22. N. F. Scherer et al., J. Chem. Phys. 95, 1487 (1991). 23. The locked frequency is $f_{L}=f_{0}-C_{0} \delta T$, where $C_{0}=$ $4.34 \mathrm{MHz} / \mathrm{ps}$ is determined by the AOPS. $\delta T$ is the delay between the arrival times of the radiofrequency wave controlling the AOPS and the laser pulse at the AOPS. Hence, $\delta T$ can be adjusted to shift the entire photon echo spectrum. The choice of $f_{0}$ is arbitrary and affects $\delta T$ accordingly. However, the locked frequency remains the same with a different choice of $f_{\mathrm{o}}$. In our experiment, we adjusted $\delta T$ and then directly measured its effect on the peak positions $\Delta f_{a}$ and $\Delta f_{b}$.

24. Other polarization signals such as the peaks with a phase dependence of $e^{i\left(\phi_{1}-\phi_{3}\right)}$ can be selected by a different 16 -step phase cycle as

$$
\begin{gathered}
\left(\rho_{X X X}-\rho_{X X \bar{X}}-\rho_{\bar{X} X X}+\rho_{\bar{X} X \bar{X}}+\rho_{Y X Y}-\rho_{\bar{Y} X Y}-\rho_{Y X \bar{Y}}\right. \\
\left.+\rho_{\bar{Y} X \bar{Y}}\right)+i\left(-\rho_{\bar{X} X \bar{Y}}+\rho_{\bar{X} X Y}-\rho_{X X Y}+\rho_{X X \bar{Y}}+\right. \\
\left.\rho_{Y X X}-\rho_{Y X \bar{X}}-\rho_{\bar{Y} X X}+\rho_{\bar{Y} X \bar{X}}\right)
\end{gathered}
$$

Hence, the 16-step phase cycle is a general approach here. For a particular polarization signal, a smaller number of phase cycles may be adequate. For example, a 10-step phase cycle can select $P_{\text {ectho }}^{(3)}$. If symbols $\mathrm{Q}, \mathrm{R}, \mathrm{S}$, and T represent a phase of $2 \pi / 5,4 \pi / 5,6 \pi / 5$, and $8 \pi / 5$, respectively; $P_{\mathrm{eccho}}^{(3)}$ is selected by

$$
\begin{gathered}
\rho 10=\left(\rho_{X X}-\rho_{X \bar{X}}+e^{-i 4 \pi / 5} \rho_{Q X}+e^{i \pi / 5} \rho_{Q \bar{X}}+e^{i 2 \pi / 5} \rho_{R X}+\right. \\
\left.e^{-i 3 \pi / 5} \rho_{R \bar{X}}+e^{-i 2 \pi / 5} \rho_{S X}\right)+e^{i 3 \pi / 5} \rho_{S \bar{X}}+e^{i 4 \pi / 5} \rho_{T X}+e^{-i \pi / 5} \rho_{T \bar{X}}
\end{gathered}
$$

25. H.-S. Tan, W. S. Warren, in preparation.

26. H.-S. Tan, personal communication.

27. W. P. de Boeij, M. S. Pshenichnikov, D. A. Wiersma, Chem. Phys. 233, 287 (1998).

28. We acknowledge the financial support from the Office of Naval Research and the New Jersey Commission on Science and Technology. We thank H.-S. Tan for pointing out the 10-step phase cycle to select $P_{\text {echo }}^{(3)}$ and L. $\mathrm{Xu}$ for his assistance in the experiment.

13 February 2003; accepted 18 April 2003 